\title{
Optical control of the spin state of two Mn atoms in a quantum dot
}

\author{
L. Besombes, ${ }^{1, *}$ C. L. Cao, ${ }^{1,2}$ S. Jamet, ${ }^{1}$ H. Boukari, ${ }^{1}$ and J. Fernández-Rossier ${ }^{2,3}$ \\ ${ }^{1}$ CEA-CNRS group "Nanophysique et semiconducteurs", Institut Néel, CNRS \& Université Joseph Fourier, \\ 25 avenue des Martyrs, 38042 Grenoble, France \\ ${ }^{2}$ Departamento de Física Aplicada, Universidad de Alicante, San Vicente del Raspeig, 03690 Spain \\ ${ }^{3}$ International Iberian Nanotechnology Laboratory, Avenida Mestre José Veiga, 4715-330 Braga, Portugal
}

(Received 15 June 2012; revised manuscript received 5 September 2012; published 5 October 2012)

\begin{abstract}
We report on the optical spectroscopy of the spin of two magnetic atoms $(\mathrm{Mn})$ embedded in an individual quantum dot interacting with a single electron, a single exciton, or a single trion. As a result of their interaction to a common entity, the Mn spins become correlated. The dynamics of this process is probed by time-resolved spectroscopy, which permits us to determine an optical orientation time in the range of a few tens of nanoseconds. In addition, we show that the energy of the collective spin states of the two Mn atoms can be tuned through the optical Stark effect induced by a resonant laser field.
\end{abstract}

DOI: 10.1103/PhysRevB.86.165306

PACS number(s): 78.67.Hc, 78.20.Jq, 78.20.Ls

\section{INTRODUCTION}

Single atom quantum devices, ${ }^{1}$ i.e., systems whose macroscopic properties depend on the quantum state of a single atom, have been demonstrated in several systems, such as single-donor Silicon transistor, ${ }^{2}$ single Mn-doped quantum dot (QD), ${ }^{3}$ a single magnetic atom on a surface, ${ }^{4}$ or a single NV center in nanodiamond. ${ }^{5}$ The manipulation of the spin state of a single atom has been shown both by optical pumping ${ }^{6-8}$ and in transport experiments. ${ }^{9}$ These systems permit us to test matter at a fundamental scale and, in some instances, have already practical applications like room temperature magnetometry with nanometer resolution. ${ }^{10} \mathrm{~A}$ controlled upscale of this primary units would permit us to test new physical phenomena and to find new applications. In this regard, the study of chains of a few magnetic atoms deposited on a metal has already given promising results along this line.${ }^{11}$ Here we report on the first step in that direction in the case of $\mathrm{Mn}$ atoms in semiconductor QDs.

When Mn atoms are included in a II-VI semiconductor QD (CdTe in $\mathrm{ZnTe}),{ }^{12}$ the spin of the optically created electronhole pair (exciton) interacts with the $5 d$ electrons of the $\mathrm{Mn}$ (total spin $S=5 / 2$ ). In the case of a singly Mn-doped QD, this leads to a splitting of the once simple photoluminescence (PL) spectrum of an individual QD into six $(2 S+1)$ components. ${ }^{3}$ Since the confined carriers and $\mathrm{Mn}$ spin wave function become strongly mixed, the optical excitation of the QD affects the spin state of the Mn through the exchange interaction offering a possibility of optical control. ${ }^{6-8,13}$

In contrast to the case of magnetic atoms in a metal, semiconductors afford the unique opportunity of controlling the exchange interaction between distant magnetic dopants by varying the carrier density of the host. This has been shown in quantum wells. ${ }^{14}$ In QDs the effect is expected to be stronger and to take advantage of the discreteness of the charge addition..$^{15,16}$ Thus, in a neutral dot containing $2 \mathrm{Mn}$ atoms, the spins are only coupled via a very short range supercharge, which would be only relevant for first or second neighbors. ${ }^{17,18}$ The injection of a single electron, whose wave function is spread along the entire dot, couples the $2 \mathrm{Mn}$ and the electron spin ferromagnetically, resulting in a ground state with $S=11 / 2$. This contrasts with the addition of a single exciton on the neutral dot, for which the Mn spins also couple ferromagnetically, but the strong spin-orbit coupling of the hole breaks spin rotational invariance. The addition of an exciton on the negatively charged dot puts the two electrons in a singlet state so that the $2 \mathrm{Mn}$ interact with a single hole.

Here we show how we can address and investigate the magnetic properties of $2 \mathrm{Mn}$ atoms, which are coupled to carriers in an individual QD. The fine structure of a confined exciton $(X)$ and negatively charged exciton $\left(X^{-}\right)$in the exchange field of the $2 \mathrm{Mn}$ atoms are analyzed and modeled in detail. We show that the $2 \mathrm{Mn}$ spins can be optically oriented by the injection of spin polarized carriers in a few tens of nanoseconds. Finally, we demonstrate that the energy of the collective spin states of the $2 \mathrm{Mn}$ atoms can be tuned by a strong resonant laser field.

\section{SAMPLES AND EXPERIMENT}

The sample used in this study is grown on a $\mathrm{ZnTe}$ substrate and contains CdTe QDs. A 6.5 monolayer thick CdTe layer is deposited at $280^{\circ} \mathrm{C}$ by atomic layer epitaxy on a ZnTe barrier grown by molecular beam epitaxy at $360^{\circ} \mathrm{C}$. The dots are formed by a Tellurium-induced process ${ }^{12}$ and protected by a 300-nm-thick ZnTe top barrier. The QDs are in the 10-nm-wide range and a few nanometers high. Mn atoms are introduced during the CdTe deposition with a density roughly equal to the density of QDs. Nonmagnetic QDs and QDs containing a low number of magnetic atoms $(1,2,3 \mathrm{Mn}, \ldots)$ are then formed.

Optical addressing of individual QDs containing magnetic atoms is achieved using micro-spectroscopy techniques. A high refractive index hemispherical solid immersion lens is mounted on the surface of the sample to enhance the spatial resolution and the collection efficiency of single-dot emission in a low-temperature $(T=5 K)$ scanning optical microscope. In PL and time-resolved optical pumping experiments, individual QDs are excited with a tunable continuous-wave dye laser tuned to an excited state of the dots. ${ }^{19}$ The resulting collected $\mathrm{PL}$ is dispersed and filtered by a 1-m double monochromator before being detected by a cooled CCD camera or a fast APD (overall time resolution $50 \mathrm{ps)}$ ). In all the experiments, a 
weak magnetic field $(B \leqslant 250 \mathrm{mT})$ can be applied in Voigt or Faraday geometry.

\section{TWO MN ATOMS IN A QUANTUM DOT}

\section{A. General considerations}

In the studied sample, the average density of Mn atoms and QDs are roughly the same. ${ }^{20}$ Since the dots do not cover all the area of the sample, a large fraction of dots have no $\mathrm{Mn}$ inside, some of them have $1 \mathrm{Mn}$, and in some rare instances, $2 \mathrm{Mn}$ and more. Dots with no $\mathrm{Mn}$, or with a $\mathrm{Mn}$ atom in the region where the exciton wave function is very small, give rise to a conventional PL spectra with 1 peak at zero field. Dots with $1 \mathrm{Mn}$ atom sufficiently coupled to the exciton give rise to a PL with six peaks at zero field, that were extensively studied before. ${ }^{3,6-8,13,19,21-26}$ The origin of the six peaks can be accounted for by the strongly anisotropic coupling of the exciton to the six electronic spin states of the Mn. To leading order, the Mn-exciton interaction results in an effective magnetic field along the growth direction given by $g \mu_{B} B_{\text {eff }}=I_{h} S_{z}$, where $S_{z}=-5 / 2, . ., 5 / 2$ are the $2 S+1=6$ projections of the Mn spin along the growth axis and $I_{h} \propto \beta\left|\Psi_{h}\left(\vec{r}_{\mathrm{Mn}}\right)\right|^{2}$ is the hole Mn exchange, which depends both on $\beta$, a material-dependent quantity, ${ }^{17}$ and on the value of the hole wave function $\Psi_{h}$ at the location $\vec{r}$ of the $\mathrm{Mn}$ in the QD. ${ }^{24}$ Typically, $I_{h} \simeq 200 \mu \mathrm{eV}$. In the case of a QD with $2 \mathrm{Mn}$, the same argument suggests that the effective exchange field felt by the exciton is now $g \mu_{B} B_{\text {eff }}=I_{h, 1} S_{z, 1}+I_{h, 2} S_{z, 2}$, which can take up to 36 different values. A full microscopic calculation ${ }^{24}$ supports this hand-waving argument. Notice that, for the 36 lines to be seen, it is important that both $I_{h, 1}$ and $I_{h, 2}$ are sizable and not too similar. Two equally coupled Mn would give rise to 11 lines. ${ }^{24}$ According to these calculations, ${ }^{24}$ the PL of a dot with more than $2 \mathrm{Mn}$ becomes already similar to those with tens of $\mathrm{Mn}$, for which it is not possible to discern a countable number of lines.

\section{B. Experimental data}

With these general considerations in mind, the observation of a dot with $2 \mathrm{Mn}$ strongly coupled to the exciton should be expected to show up as low-temperature PL spectrum with more than 6 lines but less than 36, different from broad peaks that shift strongly with an applied field, characteristic of magnetic bound polaron. ${ }^{27,28}$ Such spectra are presented in Fig. 1 for three different quantum dots. A large scale PL and the PL excitation (PLE) spectra of QD1 are displayed in the lower panel of Fig. 1. As in nonmagnetic or singly Mn-doped QDs, the emission of the $X, X^{-}$, and biexciton $\left(X^{2}\right)$ is observed simultaneously. ${ }^{25}$ Each excitonic complex, $X-2 \mathrm{Mn}, X^{-}-2 \mathrm{Mn}$, and $X^{2}-2 \mathrm{Mn}$, is split by the exchange interaction with the $2 \mathrm{Mn}$ spins.

The spin structure of a $2 \mathrm{Mn}$ QD is illustrated by the detailed spectrum of the exciton in QD1 presented in Fig. 2. From the effective field argument outlined above, the highest and lowest PL lines in the spectrum of the exciton correspond to the total spin projection of the $2 \mathrm{Mn} M_{z}=S_{z, 1}+S_{z, 2}= \pm 5$ coupled to the bright excitons $( \pm 1)$. The next lines correspond to the situation where the less coupled Mn spin has flipped its spin projection component by one unit $\left(M_{z}= \pm 4\right)$. The
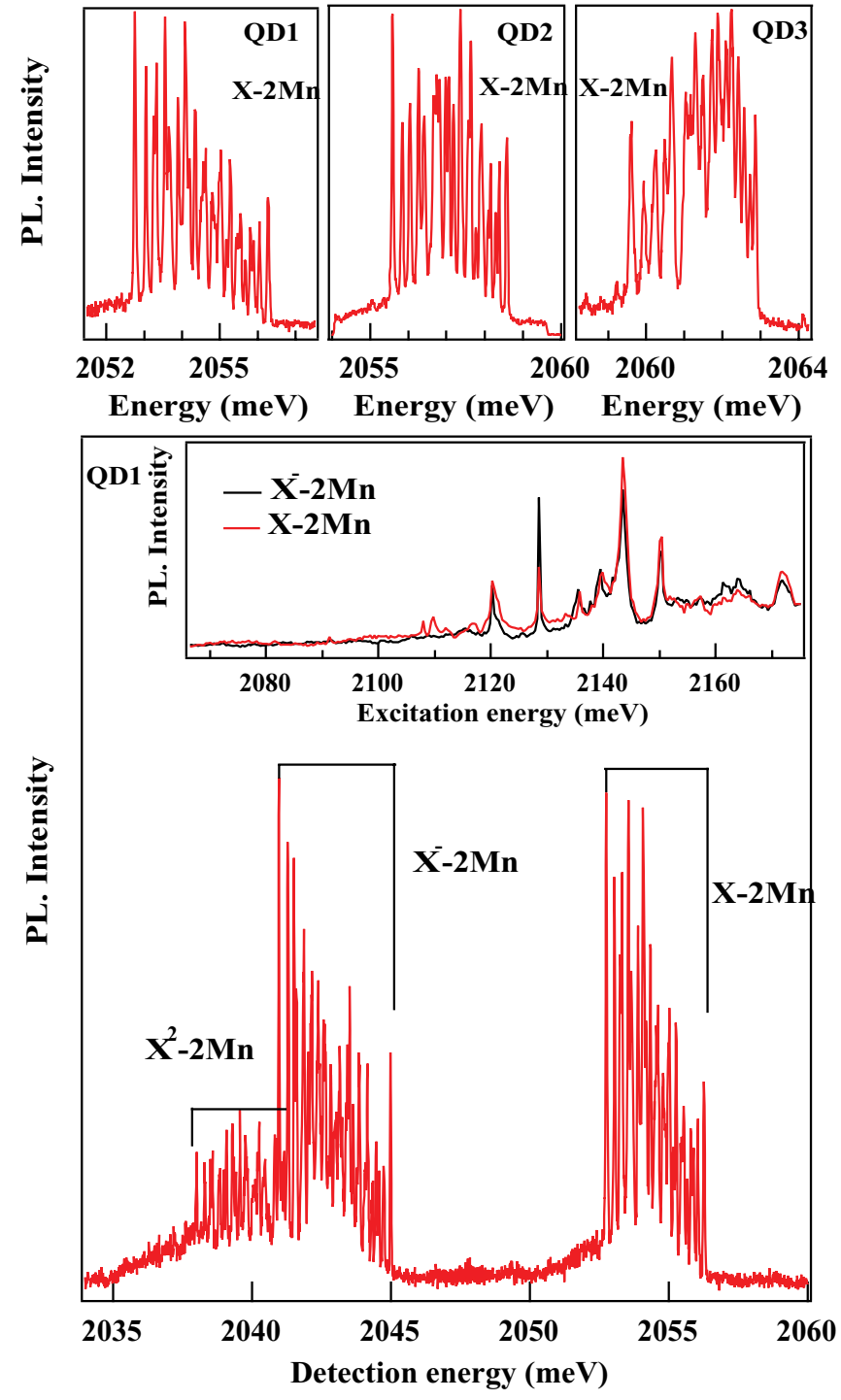

FIG. 1. (Color online) Low-temperature $(T=5 \mathrm{~K}) \mathrm{PL}$ spectrum of neutral excitons in three different $\mathrm{CdTe} / \mathrm{ZnTe}$ QDs containing $2 \mathrm{Mn}$ atoms (QD1, QD2, and QD3). The large scale spectrum of QD1 shows the coexistence of the neutral exciton $(X-2 \mathrm{Mn})$, the negatively charged exciton $\left(X^{-}-2 \mathrm{Mn}\right)$, and biexciton $\left(X^{2}-2 \mathrm{Mn}\right)$ exchanged coupled to $2 \mathrm{Mn}$ atoms. Inset: PL excitation spectra of QD1 detected on the low-energy line of $X-2 \mathrm{Mn}$ and $X^{-}-2 \mathrm{Mn}$.

emission structure becomes more complex as $M_{z}$ decreases (toward the center of the emission structure) because different configurations of the $2 \mathrm{Mn}$ spins interacting with the exciton spin are very close in energy.

\section{Model Hamiltonian}

In order to go beyond the simple effective field picture described above, we use a model Hamiltonian based on an effective spin Hamiltonian that can be derived from the $s p-d$ model for confined electrons and holes exchanged coupled to the Mn spins. ${ }^{24,29}$ The spin interacting part of the Hamiltonian of a QD describing the interaction of the electron spin $\vec{\sigma}$, the 

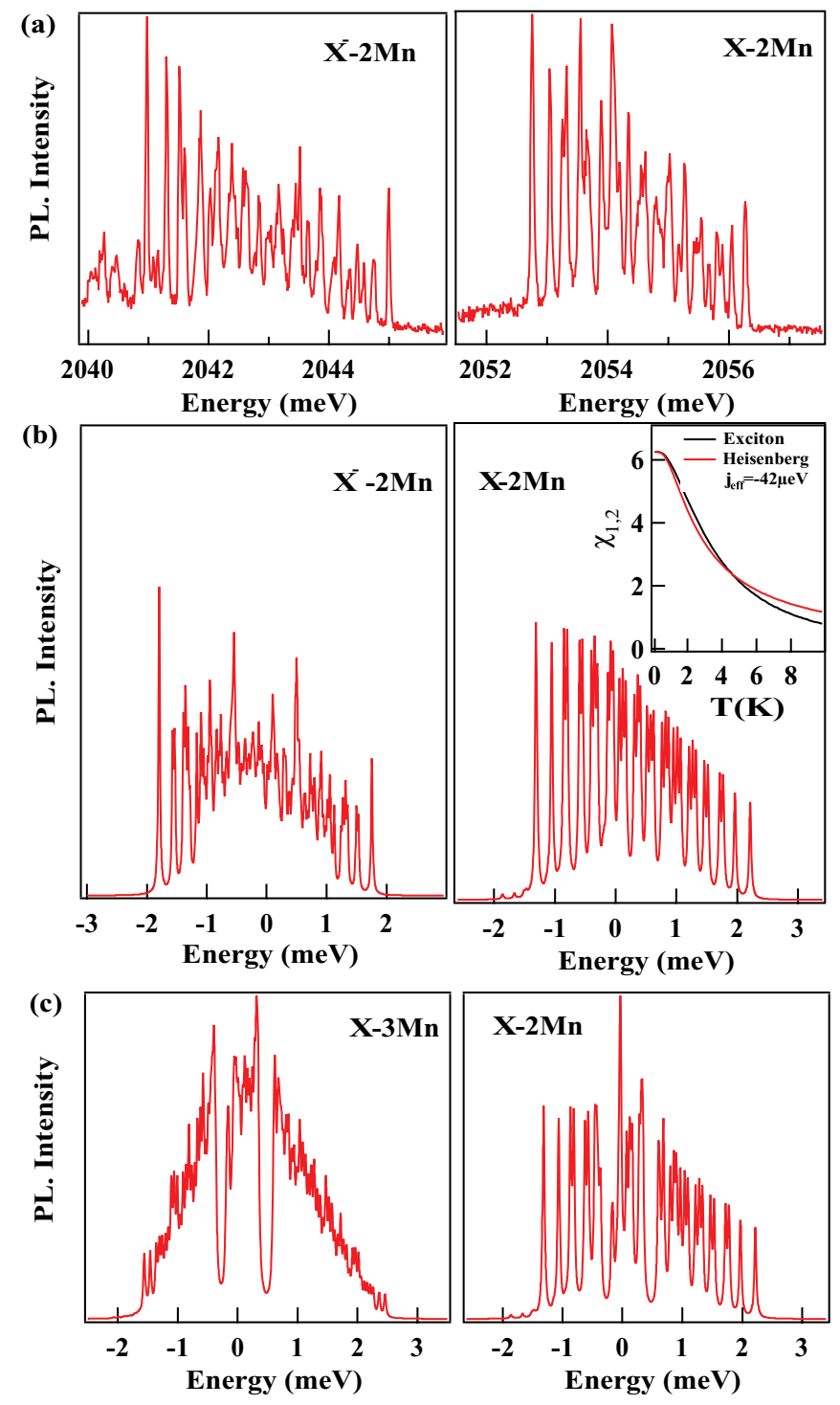

FIG. 2. (Color online) Experimental (a) and calculated (b) spectra of $X$ and $X^{-}$exchange coupled to $2 \mathrm{Mn}$ in QD1. See text for details of the calculations performed with $I_{e, 1}=-50 \mu \mathrm{eV}, I_{h, 1}=$ $150 \mu \mathrm{eV}, I_{e, 2}=-90 \mu \mathrm{eV}, I_{h, 2}=270 \mu \mathrm{eV}, I_{e h}=-600 \mu \mathrm{eV}$, an effective valence band mixing parameter $\rho / \Delta_{l h}=0.025$ and $\theta=0$. A thermalization (effective temperature $T=40 \mathrm{~K}$ ) is included for the $X-2 \mathrm{Mn}$ and hole-2Mn complexes. A broadening of the lines of $40 \mu \mathrm{eV}$ is included in the calculated spectra. The inset in (b) shows the Mn-Mn correlation function calculated for the model presented in the text (exciton) compared with the spin-spin correlation function for a Heisenberg model for two spins $S=5 / 2$. (c) The calculated spectra of $X$ coupled to 2 Mn with $\rho / \Delta_{l h}=0.1$ (right panel) and when a third $\mathrm{Mn}$ atom with $I_{e, 3}=-20 \mu \mathrm{eV}$ and $I_{h, 3}=60 \mu \mathrm{eV}$ is introduced in the QD (left panel).

hole spin $\vec{J}$, and $N$ Mn spins $\vec{S}_{n}$ reads

$$
\begin{aligned}
\mathcal{H}_{X}=\vec{\sigma} & \cdot \sum_{n=1, N} I_{e, n} \vec{S}_{n}+\vec{J} \cdot \sum_{n=1, N} I_{h, n} \vec{S}_{n}+I_{e h} \vec{\sigma} \cdot \vec{J} \\
& +\frac{1}{2} \sum_{n \neq n^{\prime}} I_{n, n^{\prime}} \vec{S}_{n} \cdot \vec{S}_{n}^{\prime} \equiv \mathcal{H}_{e M}+\mathcal{H}_{h M}+\mathcal{H}_{e h}+\mathcal{H}_{M M},
\end{aligned}
$$

where the hole spin operator, represented in the basis of the two low-energy heavy-hole states, are related to the Pauli matrices $\tau$ by $J_{z}=\frac{3}{2} \tau_{z}$ and $J_{ \pm}=\eta \tau_{ \pm}$, with

$$
\eta=-2 \sqrt{3} e^{-2 i \theta} \frac{\rho}{\Delta_{l h}},
$$

$\rho$ being the coupling energy between heavy-holes and lightholes split by the energy $\Delta_{l h}$ and $\theta$ being the angle relative to the [110] axis of the principal axis of the anisotropy responsible for the valence band mixing. ${ }^{25} I_{h, i}\left(I_{e, i}\right)$ is the exchange integral of the hole (electron) with the $\mathrm{Mn}$ atom $i, I_{e h}$ the electron-hole exchange interaction and $I_{n, n^{\prime}}$ the short ranged antiferromagnetic Mn-Mn interaction. This latter coupling is only comparable with the carrier-Mn energy when the $2 \mathrm{Mn}$ are positioned closed to each other $\left(I_{\mathrm{Mn}, \mathrm{Mn}}=0.5 \mathrm{meV}\right.$ for neighboring atoms $\left.{ }^{17,18}\right)$; it is neglected in the following calculations because the carrier-Mn interaction in the considered QD is very different for the two atoms, indicating that they are far apart.

In terms of the eigenstates of the exciton and ground state Hamiltonians, $\Psi_{X}$ and $\Psi_{G}$, the PL for a given circular polarization $\lambda= \pm$ reads: $:^{24,29}$

$$
I(\omega) \propto \sum_{G, X} P_{X}\left|\left\langle\Psi_{G}\left|\hat{p}_{\lambda}\right| \Psi_{X}\right\rangle\right|^{2} \delta\left(E_{X}-E_{G}-\hbar \omega\right),
$$

where $\hat{p}_{\lambda}$ is the operator that removes the quantum dot bright exciton that couples to the $\lambda$ photon and

$$
P_{X}=\frac{e^{-E_{X} / k_{B} T}}{\sum_{n} e^{-E_{X} / k_{B} T}}
$$

is the Boltzmann occupation function for the emitting states.

For a neutral dot, it is a good approximation to neglect the single ion anisotropy Hamiltonian and take $\Psi_{G}$ as the eigenstates of $\sum_{n=1, N} S_{z}(n)$ operator. The emission spectrum of the $X^{-}-2 \mathrm{Mn}$ can be computed using a similar spin effective Hamiltonian. ${ }^{23}$ In the ground state, the dot is described with the Hamiltonian $\mathcal{H}_{G \text {,trion }}=\mathcal{H}_{e M}+\mathcal{H}_{M M}$ and in the excited state $\mathcal{H}_{\text {ex,trion }}=\mathcal{H}_{h M}+\mathcal{H}_{M M}$, since, to first order, the two electrons form a singlet and do not couple to the Mn and hole spins. The small energy correction produced by the Mn-Mn interaction mediated by the presence of the two electrons in the initial state ${ }^{18}$ is neglected in the effective spin Hamiltonian used here.

\section{Choice of model parameters}

Calculated spectra for a neutral and negatively charged QD containing $N=2 \mathrm{Mn}$ and $N=3 \mathrm{Mn}$ are presented in Fig. 2 and compared with experiments. The main feature (i.e., overall splitting, position of PL lines, linear polarization) of the experimental spectra can be well reproduced by this spin-effective model.

The QD presented here (QD1) corresponds to a situation where the coupling with one $\mathrm{Mn}$ is about twice the coupling with the other. This can be deduced from a qualitative analysis of the structure of the low (or high) energy side of the PL spectrum of the exciton. Starting from the low energy line $\left(M_{z}= \pm 5\right)$, the second PL line, $280 \mu \mathrm{eV}$ above, corresponds to the case where the less-coupled Mn spin $\left(S_{1}\right)$ has flipped by one unit $\left(M_{z}= \pm 4\right)$. Then a group of two lines, very close in 
energy, are observed at slightly higher energy. The line that sits $560 \mu \mathrm{eV}$ above the low-energy states corresponds to $M_{z}= \pm 3$ where $S_{1}$ has flip by two units. It is very close in energy to the states $M_{z}= \pm 4$, corresponding to a spin flip by one unit of the Mn spin, which is the most coupled to the exciton $\left(S_{2}\right)$, $500 \mu \mathrm{eV}$ above the low energy state.

From this analysis of the splitting of the low-energy lines, we can estimate in the pure heavy-hole approximation (i.e., $\left.\rho / \Delta_{l h}=0\right)$ the exchange energy of the bright exciton with each Mn spin $S_{1}$ and $S_{2}: E_{S_{1}}=1 / 2\left(3 I_{h, 1}-I_{e, 1}\right) \approx 280 \mu \mathrm{eV}$ and $E_{S_{2}}=1 / 2\left(3 I_{h, 2}-I_{e, 2}\right) \approx 500 \mu \mathrm{eV}$. However, these values do not give an exact measurement of the exchange energies. Indeed, these splittings are slightly perturbed by the influence of the scattering of carriers to an excited state of the QD by the carrier-Mn coupling acting as a perturbation on the exciton wave function. ${ }^{30}$ This second-order perturbation is not included in the spin effective Hamiltonian Eq. (1) and should be taken into account in a more detailed modeling. It is responsible for the irregular spacing between the six emission lines already observed in singly Mn-doped QDs. ${ }^{21}$ Such perturbation is also present in the $2 \mathrm{Mn}$ case.

From these PL measurements at zero magnetic field, we cannot deduce the ratio $I_{h} / I_{e}$ and the electron-hole exchange interaction energy $I_{e h}$. In the model we chose typical values for $I_{h} / I_{e}$ and $I_{e h}$ measured in singly Mn-doped QDs. ${ }^{3,22}$ All the parameters used in the calculation are listed in the caption of Fig. 2. To reproduce the overall splitting of the exciton (given in the heavy hole approximation by $5\left[1 / 2\left(3 I_{h, 1}-\right.\right.$ $\left.\left.\left.I_{e, 1}\right)+1 / 2\left(3 I_{h, 2}-I_{e, 2}\right)\right]\right)$ and to match the splitting of the lines observed on both the low- and high-energy side of the exciton spectrum, we use $I_{e, 1}=-50 \mu \mathrm{eV}, I_{h, 1}=150 \mu \mathrm{eV}$, $I_{e, 2}=-90 \mu \mathrm{eV}$ and $I_{h, 2}=270 \mu \mathrm{eV}$. A good agreement is obtained with the energy distribution of the lines observed in the experimental spectra confirming the presence of $2 \mathrm{Mn}$ atoms exchanged coupled with an exciton. If a third Mn atom interacts with the exciton, $6 \times 36$ emission lines are expected. As the typical PL linewidth in CdTe/ZnTe QDs is larger than $50 \mu \mathrm{eV}$, the detail of the emission structure of such QD cannot be resolved. A broad emission line is obtained [calculated spectra presented in Fig. 2(c)] and the $3 \mathrm{Mn}$ case can be hardly distinguished from a QD containing a larger number of magnetic atoms.

To reproduce the intensity distribution in the PL spectra, a thermalization on the $X-2 \mathrm{Mn}$ levels with an effective temperature $T=40 \mathrm{~K}$ is also included in the model. The same thermalization is used for the hole-2Mn levels to reproduce the $X^{-}-2 \mathrm{Mn}$ spectrum. This effective temperature not far from the lattice temperature suggests a carrier-Mn spin relaxation time longer but close to the lifetime of the exciton (let's note that an infinite temperature would correspond to an absence of spin relaxation and an effective temperature equal to the temperature of the lattice would mean a spin relaxation much shorter than the exciton lifetime). The observed thermalization on the negatively charged exciton also traduces an efficient hole-2Mn spin relaxation during the lifetime of $X^{-}$.

Similar to the case of singly Mn-doped QDs, ${ }^{23}$ the overall splitting of the $X^{-}-2 \mathrm{Mn}$ observed experimentally is larger than the one obtained with the values of exchange integrals deduced from the modeling of the $X-2 \mathrm{Mn}$ spectra. This can be understood by the enhancement of the hole-Mn overlap (and hence of $I_{h, 1}$ and $I_{h, 2}$ ) through the increase of the Coulomb attraction of the hole by the two strongly confined electrons of $X^{-}$. A qualitative agreement is, however, obtained for the energy and intensity distribution of the PL lines on the lowand high-energy side of the charged exciton spectrum.

\section{E. Carrier induced Mn-Mn correlation}

For a given set of parameters, and a given charge state in the dot, we solve numerically the multispin Hamiltonian, obtaining eigenstates $\Psi_{X}$ and their energies $E_{X}$, and we can then compute the spin correlation function of the $2 \mathrm{Mn}$ :

$$
\chi_{1,2}\left(k_{B} T\right) \equiv\left\langle\vec{S}_{1} \cdot \vec{S}_{2}\right\rangle=\sum_{X} P_{X}\left(k_{B} T\right)\left\langle\Psi_{X}\left|\vec{S}_{1} \cdot \vec{S}_{2}\right| \Psi_{X}\right\rangle .
$$

The curve $\chi_{1,2}\left(k_{B} T\right)$ is shown in the inset of Fig. 2(b), together with the analogous curve for a Heisenberg model $j_{\text {eff }} \vec{S}_{1} \cdot \vec{S}_{2}$, for the case of correlations induced by a singleexciton. The model parameters are those of the caption of that figure. The $\chi_{12}$ for the Heisemberg model depends only on the ratio $j_{\text {eff }} / k_{B} T$ so that we take $j_{\text {eff }}$ as the one that minimize the difference between the two correlation curves. This procedure permits us to extract an energy to characterize the indirect Mn-Mn correlation.

When we use this procedure for the case of 1 electron Heisenberg coupled to $2 \mathrm{Mn}$, we find $j_{\text {eff }}=-6 \mu \mathrm{eV}$, for the parameters given in the caption of Fig. 2 and a scaling of $j_{\text {eff }} / \sqrt{I_{e, 1} I_{e, 2}} \simeq-0.05$, in agreement with the effective coupling derived by one of us. ${ }^{15}$ For the same parameters, in the case of the exciton-mediated effective interaction, we find $j_{\text {eff }}=-42 \mu \mathrm{eV}$ and a scaling $j_{\text {eff }} / \sqrt{I_{h, 1} I_{h, 2}} \simeq-0.2$.

\section{F. Valence band mixing and optical anisotropy}

Figure 3 reveals the linear polarization rate, which is observed in the center of the $X-2 \mathrm{Mn}$ and $X^{-}-2 \mathrm{Mn}$ PL spectra of QD1. This optical polarization can be explained by the presence of a small valence band mixing induced either by a shape or a strain anisotropy in the QD plane.

In the case of the exciton, a valence band mixing combined with the short-range electron-hole exchange interaction $I_{e h}$, couples the bright excitons +1 and -1 associated with the same Mn spins configurations (fixed $M_{z}$ ). ${ }^{25}$ As in nonmagnetic QDs, this mixing creates linearly polarized eigenstates. This mechanism is more efficient in the center of the $X-2 \mathrm{Mn} \mathrm{PL}$ structure where $M_{z}$ is small and the +1 and -1 excitons coupled with the Mn spins close in energy (right panels of Fig. 3).

In the absence of electron-hole exchange interaction (situation of a negatively charged QD) the valence band mixing allows simultaneous hole-Mn flip-flops. For a single Mn, this coupling fully mixes the states $\left|J_{z}=+3 / 2, S_{z}=-1 / 2\right\rangle$ and $\left|J_{z}=-3 / 2, S_{z}=+1 / 2\right\rangle$, which are degenerate in the absence of valence band mixing. This gives rise to fully linearly polarized lines in the center of the charged excitons PL spectra. ${ }^{23}$ In the $2 \mathrm{Mn}$ case, the states involved in a hole-Mn flip-flop cannot be degenerate. However, the mixing is still significant if the states are close in energy. This is the case for the $\mathrm{Mn}$ spins states $S_{z, 1}= \pm 1 / 2$ and $S_{z, 2}= \pm 1 / 2$ (total $\operatorname{spin} M_{z}=0$ or \pm 1 , which are in the center of the hole-Mn energy levels 


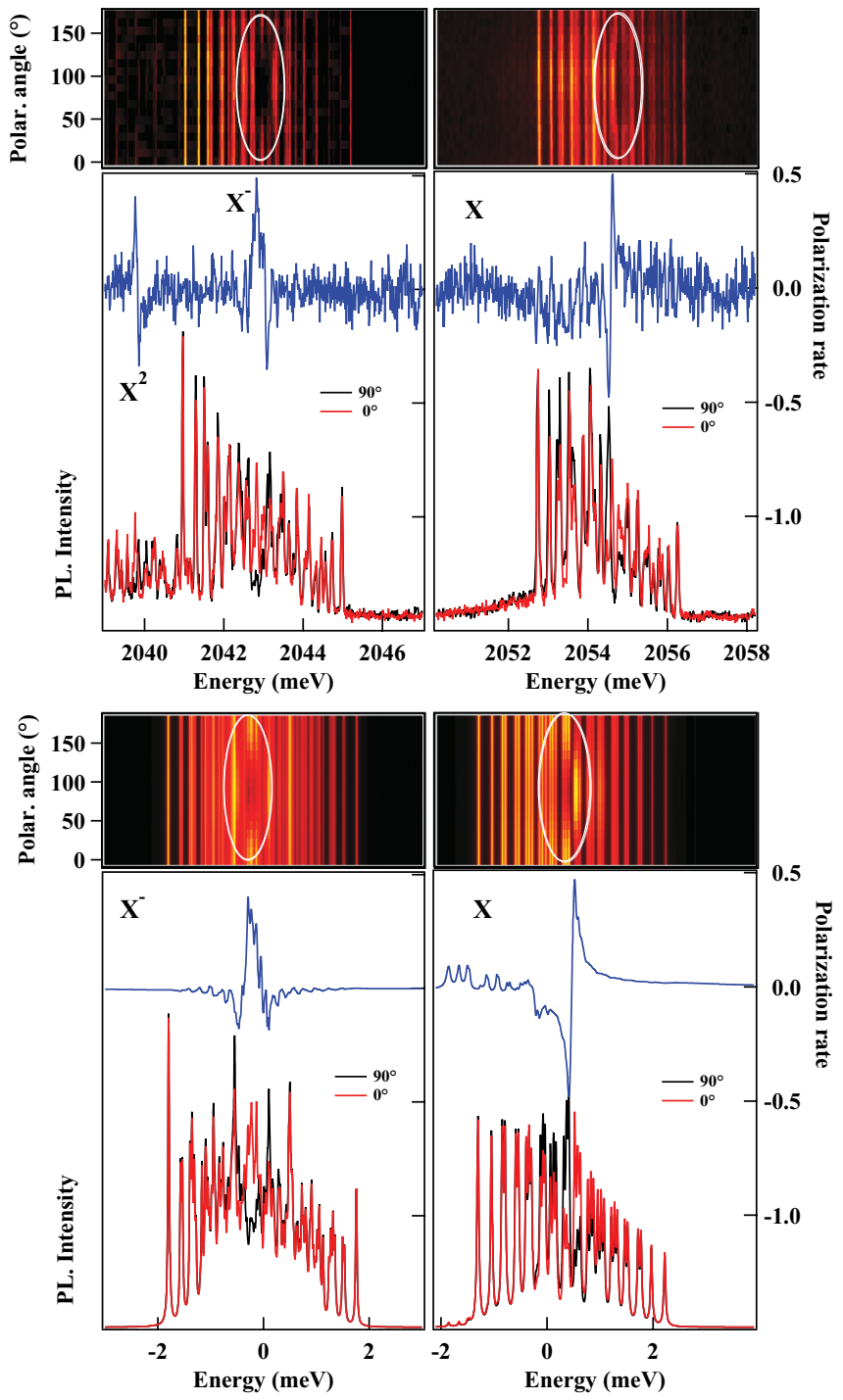

FIG. 3. (Color online) Top panel: experimental linear polarization dependence of the PL intensity of $X$ and $X^{-}$exchange coupled to $2 \mathrm{Mn}$ atoms (QD1). The polarization angle is relative to the [110] direction. The PL are presented for orthogonal linear analyzer directions (red and black curves) and the linear polarization rate is displayed in blue. Bottom panel: calculated linear polarization dependence of the PL intensity of $X$ and $X^{-}$with the same parameters as those described in the legend of Fig. 2.

distribution. The flip-flops couple together these different states of the hole-2Mn complex in the initial state of the negatively charged exciton optical transitions. These mixed states are at the origin of the partially linearly polarized lines in the center of the spectra of the charged exciton (left panels of Fig. 3).

The linear polarization rate observed experimentally is well reproduced by the spin effective model [Fig. 3 (bottom panel)] for both the $X-2 \mathrm{Mn}$ and $X^{-}-2 \mathrm{Mn}$ if a weak effective valence band mixing $\left(\rho / \Delta_{l h}=0.025\right)$ is introduced in the spin effective Hamiltonian. For large valence band mixing (with typically $\rho / \Delta_{l h}>0.1$, which is often the case in CdTe/ZnTe QDs), the valence band mixing also permits direct coupling between bright and dark excitons through a hole-Mn spin flip. The model shows [Fig. 2(c)] that in the 2 Mn case, such valence band mixing perturbs the low-energy side of the exciton emission spectrum. Additional PL lines mixed with the bright exciton levels and energy gaps appear giving rise to a more complex emission structure difficult to accurately compare with experiments (case of QD3).

\section{OPTICAL CONTROL OF THE SPIN STATE OF TWO MN ATOMS}

Earlier work has shown that optical pumping of the QD with circularly polarized light can efficiently control the occupation of the Mn spin states in the case of a single Mn QD. ${ }^{6-8} \mathrm{We}$ show here the same concept, i.e., that the $2 \mathrm{Mn}$ spins can be optically oriented by the injection of spin polarized carriers. To optically pump the Mn spins, the QD is excited with a tunable continuous wave laser on resonance with the excited state corresponding to the strong PLE peak at $2143.5 \mathrm{meV}$ (inset of Fig. 1). ${ }^{19}$ The relative intensity of the PL lines of the
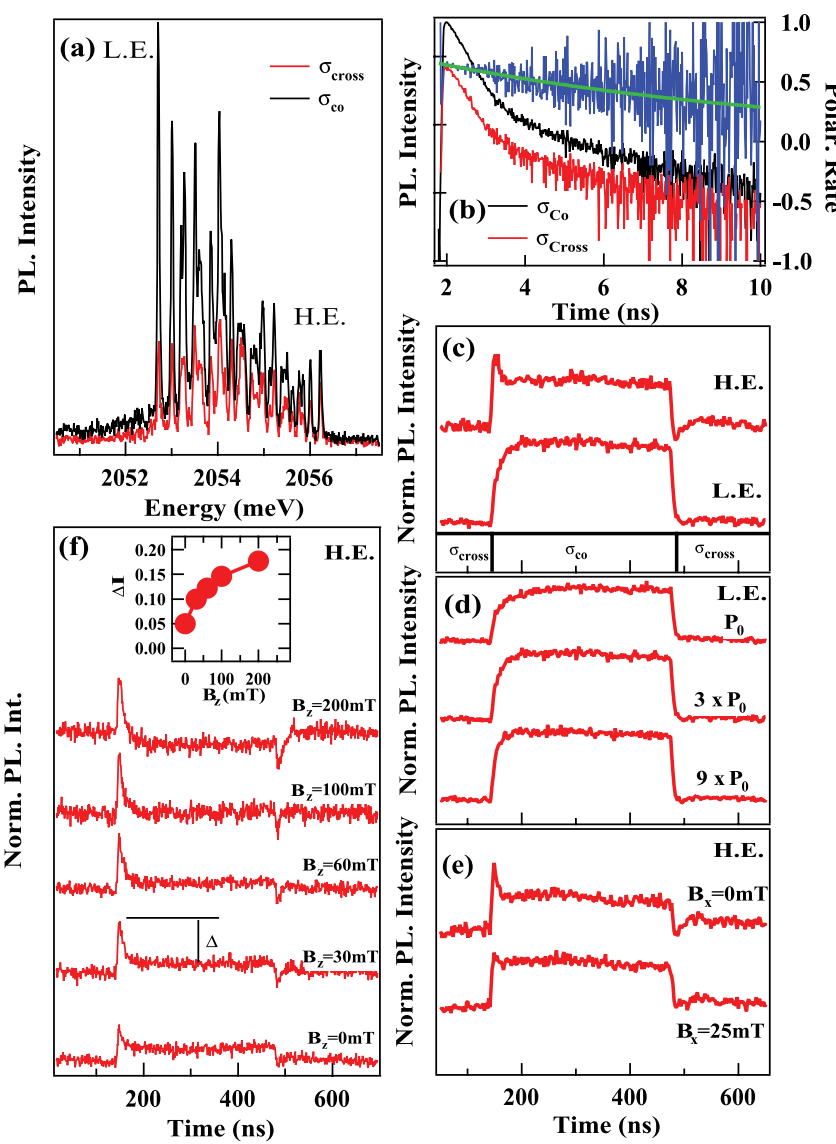

FIG. 4. (Color online) (a) PL of $X-2 \mathrm{Mn}$ in QD1 under circularly polarized excitation on an excited state (peak at $2143.5 \mathrm{meV}$ in the inset of Fig. 1) and detected in co- and cross-circular polarization. (b) Time-resolved polarization rate of the low energy line (L.E.) under pulsed excitation at $2143.5 \mathrm{meV}$. (c) Optical orientation transients under polarization switching of the excitation detected in circular polarization on the low (L.E.) and high (H.E.) energy lines. (d) Excitation intensity dependence of the spin transients detected on the low-energy line. (e) and (f) The magnetic field dependence of the spin transients in Faraday (e) and Voigt (f) configuration detected on the high-energy line. 
$X$-2Mn state depends strongly on the correlation between the polarization of the excitation and detection [Fig. 4(a)]. As each line corresponds to a given spin state of the $2 \mathrm{Mn}, M_{z}$, this shows that the whole process of spin injection and relaxation creates a nonequilibrium distribution of the Mn spin states.

In opposition to the observation in QDs containing a large number of magnetic atoms, ${ }^{28}$ the spin polarization of the exciton coupled to the $2 \mathrm{Mn}$ is well conserved during its lifetime. Nevertheless, a bright exciton spin relaxation time of about $10 \mathrm{~ns}$ can be extracted from the time decay of the circular polarization rate of the exciton observed under pulsed ( 2 ps pulses) quasiresonant excitation [exponential fit in Fig. 4(b)]. The biexponential decay observed in the PL transients also suggest an efficient transfer from dark to bright excitons involving either an electron or a hole spin flip. ${ }^{26}$

The spin relaxation of the coupled carriers and Mn spins can be exploited for an optical orientation of the Mn spins. The main features of time resolved optical orientation experiments are reported in Figs. 4(c)-4(f). In these experiments, the polarization of the continuous wave excitation laser is modulated between two circular states by an electro-optic modulator with a rise time of $10 \mathrm{~ns}$ combined with a quarter-wave plate. ${ }^{6,8}$ Switching the circular polarization of the laser produces a change of the PL intensity with two transients [Fig. 4(c)]: first, an abrupt one, reflecting the population change of the spin-polarized excitons; then a slower transient with opposite signs on the two extreme PL lines (i.e., when monitoring the Mn spin states $M_{z}=+5$ or $M_{z}=-5$ ) [Fig. 4(c)] and a characteristic time which is inversely proportional to the pump intensity [Fig. 4(d)]. This is the signature of an optical orientation of the spin of the $2 \mathrm{Mn}$ atoms. This orientation takes place in a time-scale of a few tens of nanoseconds, depending on the laser excitation intensity.

As shown in Fig. 4(f), the efficiency of the spin orientation increases as soon as a magnetic field, $B_{z}$, of a few tens of millitesla is applied along the QD growth axis. By contrast, an in-plane magnetic field $\left(B_{x}\right)$ induces coherent precession of the Mn spins away from the optical axis (QDs growth axis), so that the average spin polarization, probed by the amplitude of the optical pumping signal, decays. A transverse field of about $25 \mathrm{mT}$ is enough to erase the optical pumping [Fig. 4(e)]. As already demonstrated for individual $\mathrm{Mn}$ atoms, the magnetic field dependence of the optical pumping is due to the fine structure of the Mn controlled by the local strain environment of the atom. ${ }^{6}$ The timescale of the pumping of the $2 \mathrm{Mn}$ as well as its magnetic field dependence are similar to what was observed for individual Mn atoms. ${ }^{6,8}$

Finally, it is shown in Fig. 5 that, in QDs with a weak valence band mixing (QD1), a selective addressing of the $X-2 \mathrm{Mn}$ complex can be achieved with a control laser on resonance with one of the PL line and the energy of the $2 \mathrm{Mn}$ spins tuned optically. ${ }^{13}$ When a control single mode laser is tuned to the high energy line of $X-2 \mathrm{Mn}$ in $\sigma+$ polarization, one observes a power-dependent [Fig. 5(b)] and tuning-dependent [Fig. 5(c)] splitting of the low energy line of $X-2 \mathrm{Mn}$ in $\sigma-$ polarization. Since the ground state is the same for both transitions, this shows that the energy of Mn spins of the ground state are tuned by the resonant laser field.

The strong electric field of the control laser field mixes the states with a $2 \mathrm{Mn}$ spins component $M_{z}=+5$ in the

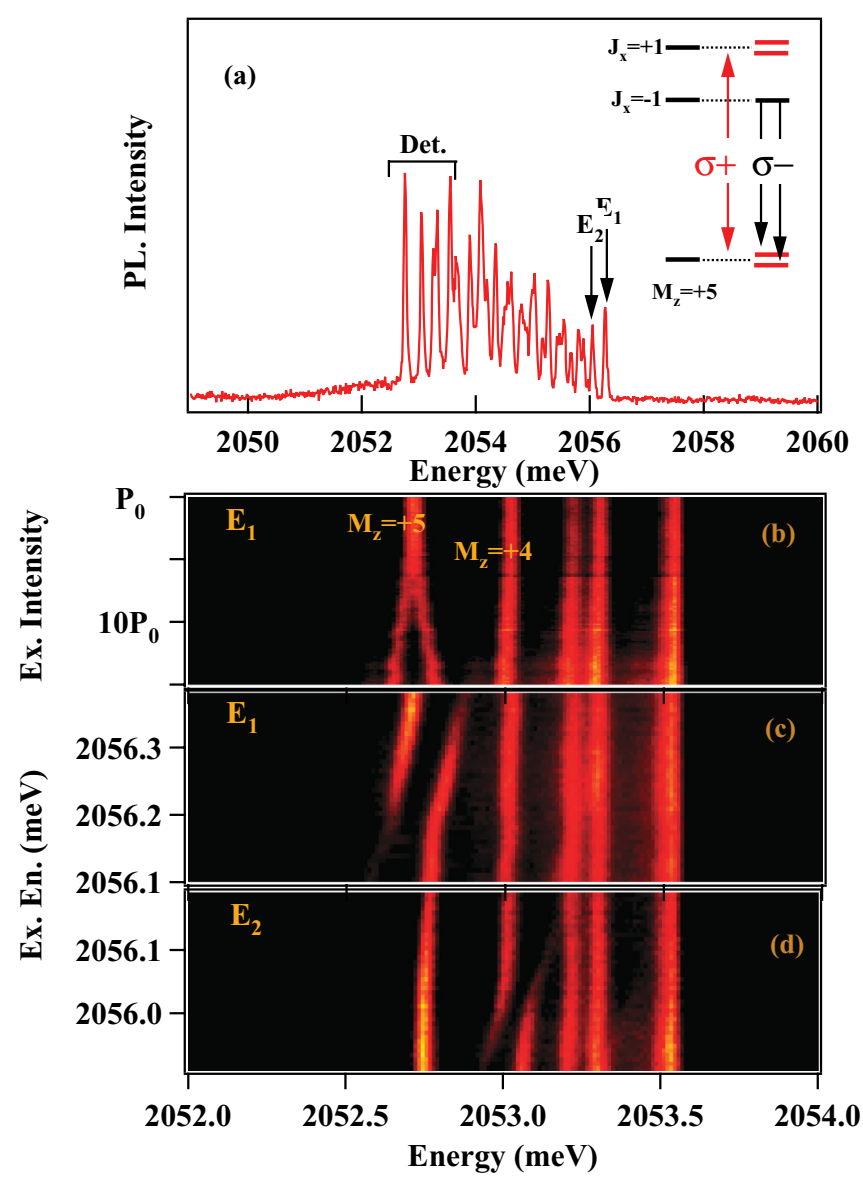

FIG. 5. (Color online) (a) Nonresonant PL of $X-2 \mathrm{Mn}$ in QD1. The inset illustrates the effect of a $\sigma+$ resonant laser field on $M_{z}=+5$ $\left(E_{1}\right)$. (b) PL intensity map of the $X-2 \mathrm{Mn}$ versus the power of the control laser in resonance with $E_{1}$. (c, d) PL maps of the $X-2 \mathrm{Mn}$ when the control laser energy is tuned around $E_{1}(\mathrm{c})$ and $E_{2}(\mathrm{~d})$.

presence $(X-2 \mathrm{Mn})$ or absence (2 Mn alone) of the exciton. At resonance, hybrid matter-field states are created [Fig. 5(b)]. As the laser detuning increases, the optically active transitions asymptotically approach the original excitonic transitions where the remaining energy offset is the optical Stark shift [Fig. 5(d)]. The use of a resonant laser field allows us to individually address any spin state of the $2 \mathrm{Mn}$ if the corresponding excitonic transition is sufficiently isolated from the others [Fig. 5(c) $M_{z}=+5$ and Fig. 5(d) $M_{z}=+4$ ]. The spin-dependent strong coupling with the laser field modifies the Mn fine structure (interaction with the crystal field) and the hyperfine structure (interaction with the ${ }^{55} \mathrm{Mn}$ nuclei). This optically controlled energy shift should affect the spin dynamics of the two magnetic atoms.

\section{CONCLUSION}

In summary, we demonstrated the possibility to access the spin states of two Mn atoms embedded in a CdTe/ZnTe QD. The two spins can be optically oriented and their energy tuned by a resonant laser field. As each of these spins is exchanged coupled to the confined carriers, one would expect to be able to control their mutual interaction. In future experiments, each Mn spin could be individually addressed by a resonant 
microwave excitation while their coupling could be turned on and off by the controlled injection of an individual carrier.

\section{ACKNOWLEDGMENTS}

This work is supported by the French ANR Contract QuAMOS, the Fondation Nanoscience (RTRA Grenoble), and the EU ITN Project Spin-Optronics. J.F.R. acknowledges funding from Ministerio de Economia, Grants No. FIS201021883-C02-01, Plan Nacional de I+D, codigo FIS No. FIS2010-21883-C02-01, and CONSOLIDER No. CSD20070010, and from Generalitat Valenciana, Grant Prometeo 2012-011. *lucien.besombes@grenoble.cnrs.fr

${ }^{1}$ P. M. Koenraad and M. E. Flatte, Nat. Mater. 10, 91 (2011).

${ }^{2}$ M. Fuechsle, J. A. Miwa, S. Mahapatra, H. Ryu, S. Lee, O. Warschkow, L. C. L. Hollenberg, G. Klimeck, and M. Y. Simmons, Nature Nanotech. 7, 242 (2012).

${ }^{3}$ L. Besombes, Y. Leger, L. Maingault, D. Ferrand, H. Mariette, and J. Cibert, Phys. Rev. Lett. 93, 207403 (2004).

${ }^{4}$ C. Hirjibehedin, C.-Y. Lin, A. F. Otte, M. Ternes, C. P. Lutz, B. A. Jones, and A. J. Heinrich, Science 317, 1199 (2007).

${ }^{5}$ F. Jelezko, T. Gaebel, I. Popa, A. Gruber, and J. Wrachtrup, Phys. Rev. Lett. 92, 076401 (2004).

${ }^{6}$ C. Le Gall, L. Besombes, H. Boukari, R. Kolodka, J. Cibert, and H. Mariette, Phys. Rev. Lett. 102, 127402 (2009).

${ }^{7}$ M. Goryca, T. Kazimierczuk, M. Nawrocki, A. Golnik, J. A. Gaj, P. Kossacki, P. Wojnar, and G. Karczewski, Phys. Rev. Lett. 103, 087401 (2009).

${ }^{8}$ C. Le Gall, R. S. Kolodka, C. Cao, H. Boukari, H. Mariette, J. Fernandez-Rossier, and L. Besombes, Phys. Rev. B 81, 245315 (2010).

${ }^{9}$ S. Loth, K. von Bergmann, M. Ternes, A. F. Otte, C. P. Lutz, and A. J. Heinrich, Nature Physics 6, 340 (2010).

${ }^{10}$ P. Maletinsky, S. Hong, M. S. Grinolds, B. Hausmann, M. D. Lukin, R. L. Walsworth, M. Loncar, and A. Yacoby, Nature Nanotech. 7, 320 (2012).

${ }^{11}$ S. Loth, S. Baumann, C. P. Lutz, D. M. Eigler, and A. J. Heinrich, Science 335, 196 (2012).

${ }^{12}$ P. Wojnar, C. Bougerol, E. Bellet-Amalric, L. Besombes, H. Mariette, and H. Boukari, J. Cryst. Growth 335, 28 (2011).

${ }^{13}$ C. Le Gall, A. Brunetti, H. Boukari, and L. Besombes, Phys. Rev. Lett. 107, 057401 (2011).

${ }^{14}$ H. Boukari, P. Kossacki, M. Bertolini, D. Ferrand, J. Cibert, S. Tatarenko, A. Wasiela, J. A. Gaj, and T. Dietl, Phys. Rev. Lett. 88, 207204 (2002).
${ }^{15}$ J. Fernandez-Rossier and L. Brey, Phys. Rev. Lett. 93, 117201 (2004).

${ }^{16}$ F. Qu and P. Hawrylak, Phys. Rev. Lett. 95, 217206 (2005).

${ }^{17}$ J. Furdyna, J. Appl. Phys. 64, R29 (1988).

${ }^{18}$ F. Qu and P. Hawrylak, Phys. Rev. Lett. 96, 157201 (2006).

${ }^{19}$ M. M. Glazov, E. L. Ivchenko, L. Besombes, Y. Leger, L. Maingault, and H. Mariette, Phys. Rev. B 75, 205313 (2007).

${ }^{20}$ L. Maingault, L. Besombes, Y. Léger, C. Bougerol, and H. Mariette, Appl. Phys. Lett. 89, 193109 (2006).

${ }^{21}$ L. Besombes, Y. Leger, L. Maingault, D. Ferrand, H. Mariette, and J. Cibert, Phys. Rev. B 71, 161307 (2005).

${ }^{22}$ Y. Leger, L. Besombes, L. Maingault, D. Ferrand, and H. Mariette, Phys. Rev. B 72, 241309(R) (2005).

${ }^{23}$ Y. Leger, L. Besombes, J. Fernandez-Rossier, L. Maingault, and H. Mariette, Phys. Rev. Lett. 97, 107401 (2006).

${ }^{24}$ J. Fernandez-Rossier, Phys. Rev. B 73, 045301 (2006).

${ }^{25}$ Y. Leger, L. Besombes, L. Maingault, and H. Mariette, Phys. Rev. B 76, 045331 (2007).

${ }^{26}$ L. Besombes, Y. Leger, J. Bernos, H. Boukari, H. Mariette, J. P. Poizat, T. Clement, J. Fernandez-Rossier, and R. Aguado, Phys. Rev. B 78, 125324 (2008).

${ }^{27}$ T. Clement, D. Ferrand, L. Besombes, H. Boukari, and H. Mariette, Phys. Rev. B 81, 155328 (2010).

${ }^{28}$ L. Klopotowski, L. Cywinski, P. Wojnar, V. Voliotis, K. Fronc, T. Kazimierczuk, A. Golnik, M. Ravaro, R. Grousson, G. Karczewski, and T. Wojtowicz, Phys. Rev. B 83, 081306(R) (2011).

${ }^{29}$ C. L. Cao, L. Besombes, and J. Fernandez-Rossier, Phys. Rev. B 84, 205305 (2011).

${ }^{30}$ A. H. Trojńar, M. Korkusinski, E. S. Kadantsev, P. Hawrylak, M. Goryca, T. Kazimierczuk, P. Kossacki, P. Wojnar, and M. Potemski, Phys. Rev. Lett. 107, 207403 (2011). 